ESTRATÉGIA 


\title{
AMPLIANDO A MENSURAÇÃO DA INTENÇÃO EMPREENDEDORA
}

\author{
EXPANDING MEASUREMENT OF ENTREPRENEURIAL INTENT
}

Roosiley dos Santos Souza

Universidade Federal de Mato Grosso do Sul

Amelia Silveira

Universidade Federal de Santa Catarina

Sabrina do Nascimento

Universidade Federal de Santa Catarina

\author{
Data de submissão: $0 \mid$ abr. 20 |7. Data de aprovação: \\ 20 out. 2017. Sistema de avaliação: Double blind review. \\ Universidade FUMEC / FACE. Prof. Dr. Henrique Cordeiro \\ Martins. Prof. Dr. Cid Gonçalves Filho.
}

\section{RESUMO}

A Teoria do Comportamento Planejado (TCP) é reconhecida como uma teoria de base para a intenção empreendedora (IE). E o Questionário de Intenção Empreendedora (EIQ) tem sido adotado para mensurar a IE em universidades. Buscando ampliar o estudo sobre a mensuração da IE, analisou-se a IE com base na mediação da Atitude Pessoal (AP) em relação à IE, no que tange às relações com as Normas Subjetivas (NS) e com o Controle do Comportamento Percebido (CCP). Utilizaram-se procedimentos estatísticos descritivos e de Structural Equation Modeling (SEM), com mínimos quadrados parciais e modelo de caminhos PLS Path Modeling (PLS-PM). Estudantes de graduação, em duas universidades federais brasileiras, foram os respondentes. Os resultados permitiram comprovar que a AP é uma variável forte, que se fortalece em $17 \%$ a mais neste estudo, confirmando hipóteses de que CCP influencia significativamente AP; a AP tem papel mediador na relação entre CCP e IE, e para a relação NS e IE. Essa relação entre CCP e IE e de NS e IE sofre influência de AP.

\section{PALAVRAS-CHAVE}

Intenção empreendedora. Teoria do Comportamento Planejado. Universidades Federais Brasileiras. Estudantes Universitários. 


\section{ABSTRACT}

The Theory of the Planned Behavior (TPB) is known as a base theory for the entrepreneurial intention (EI). The Entrepreneurial Intention Questionnaire (EIQ) has been adopted to measure the El. Seeking to expand the study on the measurement of IE, complementing the validation of the EIQ, IE was analyzed based on the mediation of Personal Attitude (PA) in relation to the El, regarding the relations with Subjective Norms (SN) and with Perceived Behavior Control (PBC). We used descriptive statistical procedures and Structural Equation Modeling (SEM), with partial least squares and PLS Path Modeling (PLS-PM) path model. Graduating students, in two Brazilian federal universities, were the respondents. The results allowed proving that the PA is a strong variable, which is strengthened by an additional I $7 \%$ in this study, confirming the hypothesis that PBC influences significantly PA; the PA has a mediating role for the relationship between $P B C$ and El, and $S N$ and El.This relationship between $P B C$ and $E I$ and $S N$ and $E I$ is influenced by $A P$.

\section{KEYWORDS}

Entrepreneurial Intention. Theory of the Planned Behavior. Brazilian Federal Universities. University Students.

\section{INTRODUÇÃO}

A Intenção Empreendedora (IE) se volta para o estudo da predição e da propensão do indivíduo em se tornar um empreendedor e fundamenta-se, principalmente, na Teoria do Comportamento Planejado (TCP), de Ajzen (I99|). A TCP prevê que o comportamento de agir resulta da combinação de três atitudes: a Atitude Pessoal (AP) diante desse comportamento, as Normas Subjetivas (NS) que lhes são implícitas, e a percepção sobre o Controle do Comportamento Percebido (CCP), em causa. Assim, o modelo de Ajzen (I99I) compreende três variáveis independentes que precedem a formação da intenção e que preveem o comportamento humano. A primeira variável AP permite determinar o momento favorável para uma ação. A se- gunda variável NS indica a percepção que um indivíduo tem sobre a comunidade que o rodeia, que influencia e determina seu comportamento. E a terceira variável CCP reflete o grau de percepção do encontrado, levando o indivíduo a ter também um determinado comportamento. Com esse entendimento, os comportamentos previstos são intencionais e previstos pela intenção existente para esse comportamento. A intencionalidade é fundamentada na psicologia cognitiva, precedendo determinado comportamento humano. Essa TCP proposta por Ajzen (1991) tem sido citada e reconhecida desde sua publicação em obras como a de Krueger e Carsud (1993) como base para os estudos de IE, no campo do empreendedorismo. Em período mais recente, com o objetivo 
de estudar as teorias dominantes, as limitações e as perspectivas da TCP na área de IE, diversos trabalhos de meta-análises e de revisão de literatura contribuíram para alicerçar esse entendimento. (LIÑÁN; MORIANO, 20 I4; SCHLAEGEL; KOENIG, 20।4; QIAN et al, 20I4; FAYOLLE; LIÑÁN, 2015; KAUTONEN; GELDEREN; FINK, 20I5; LIÑÁN; FAYOLLE, 20I5; LORTIE; CASTOGIOVANNI, 20I5; RUEDA; MORIANO; LIÑÁN, 20I5; SANTOS; MARTINS; SILVEIRA, 20 I6; SILVEIRA et al, 2017 ; TERJESEN; HESSELS; LI, 20I6). A obra de Ajzen (I99I) pode ser considerada, assim, uma obra seminal, uma vez que vem sendo citada e revisada como um modelo teórico que vem se apresentando como satisfatório, apresentando predominância sobre os demais. Essa teoria volta-se para prever e explicar o comportamento humano, em contextos específicos.

Por sua vez, para mensurar a IE de estudantes universitários em diferentes contextos mundiais há indicação de que um instrumento de coleta de dados que tem sido adotado com maior frequência. Este instrumento, denominado Questionário de Intenção Empreendedora (EIQ), constitui-se de uma escala psicométrica, que toma como base os pressupostos de Ajzen (I99I) e a estrutura da TCP, tendo sido adotado no estudo desenvolvido por Liñán e Chen (2009), hoje um dos mais citados na literatura do tema. Dois anos mais tarde, continuando os estudos sobre o EIQ, foi realizada uma revisão e atualização da escala psicométrica por Liñán, Urbano e Guerrero (20l I), sendo que buscaram sua melhor adequação como instrumento de coleta de dados para mensurar a intenção empreendedora, em diferentes contextos, com estudantes universitários.
A IE se constitui assim, como área de estudo em desenvolvimento, na qual a TCP de Ajzen (1991) e o estudo de Liñán, Urbano e Guerrero (20II) se apresentam, atualmente, como básicos e adequados para amparar pesquisas de IE, com estudantes de universidades. Cabe ressaltar, como base no que afirma o estudo de Rueda, Moriano e Liñán (20I5), que essas pesquisas de IE, com estudantes universitários, têm sido realizadas em inúmeros países. Recentemente se pode citar trabalhos realizados em vários países, como os de Zhang, Duysters e Cloodt (20I4), na China; Kautonen, Gelderen e Fink (20I5), na Áustria e Finlândia; Debarliev et al. (20I5), na República da Macedônia; Pfeifer, Šarlija e Zekić Sušac (20 I6), na Croácia; Nabi et al., (20 I6), na Inglaterra; Kim-Soon, Ahmad, Ibrahim (20 l6), na Malasia; Al-Jubari, Hassan e Hashim (20।7), no Yemen; Utami (20l7), na Indonesia; Mwiya (20l7), em Zâmbia, na Africa; e Zampetakis et al. (20l8), na Grécia, apenas para citar alguns. No Brasil, entre os mais atuais, estão os de Lovison (20l4), Fontenele, Brasil e Sousa (2015), Souza (20I5), Souza e Silveira (20l6), Souza, Silveira e Nascimento (2017), Vasconcelos et al. (2017).A maioria desses autores adotou em seus estudos o ElQ, total ou parcialmente, seguindo os fundamentos da TCP de Ajzen (1991) e dos estudos de Liñán e Chen (2009) e/ou de Liñán, Urbano e Guerrero (20l I). Em tais estudos a amostra de estudantes universitários foi comum na investigação de intenção empreendedora, visto que oferece a vantagem de idade e qualificações semelhantes, tornando a amostra mais homogênea (LIÑÁN, 2008). Dessa forma, tanto a TCP de Ajzen (I99I) quanto o ElQ de Liñán, Urbano e Guerrero (20II), servem de modelo para am- 
parar pesquisas de intenções empreendedoras em universidades federais brasileiras com alunos de cursos de graduação, uma vez que têm sido adotados em estudos anteriores e internacionais, apresentando resultados com similitudes.

Segundo Terjesen, Hessels e Li (2016), entretanto, essa literatura, apesar de crescente, ainda carece de visões ampliadas que complementem o entendimento sobre o modelo teórico da TCP e de suas três variáveis (atitude pessoal dos discentes em relação à IE, no que se refere às relações com as Normas Subjetivas e com o Controle do Comportamento Percebido, e da mensuração da intenção empreendedora. Esta também é a visão de Pfeifer, Šarlija e Zekić Sušac (2016) e de Nabi, Walmsley, Liñán, Akhtar e Neame (2018), que incentivam a natural superação de limitações identificadas nos estudos do tema, com estudos complementares.

Seguindo essa tendência, e compartiIhando do entendimento, a questão de pesquisa que norteia o presente estudo se volta para a seguinte indagação: até que ponto a testagem do papel mediador da Atitude Pessoal dos discentes em relação à Intenção Empreendedora, no que se refere às relações com as Normas Subjetivas e com - Controle do Comportamento Percebido, pode contribuir para ampliar o entendimento sobre a intenção empreendedora? Com esta perspectiva, o estudo teve como objetivo mediar a Atitude Pessoal em relação à Intenção Empreendedora, no que tange às relações com as Normas Subjetivas e com o Controle do Comportamento Percebido. Para tanto, os seguintes objetivos específicos foram definidos:

a) mensurar a intenção empreendedora, com a adoção do EIQ, de alunos que cursam graduação em Administração e em Engenharia de Produção na Universidade Federal de Mato Grosso do Sul (UFMS) e na Universidade Federal de Dourados (UFGD), ambas localizadas no Estado do Mato Grosso do Sul, Brasil;

b) testar o papel mediador da atitude pessoal destes alunos em relação à Intenção Empreendedora referente às relações com as Normas Subjetivas e com o Controle do Comportamento Percebido;

c) propor um modelo estrutural para ampliar o entendimento sobre intenção empreendedora.

Justifica-se 0 interesse pelas universidades UFMS e da UFGD uma vez que essas se configuram como duas instituições de ensino superior federais brasileiras, tradicionais e representativas para o desenvolvimento em uma região considerada nova, elevada à categoria de estado em 1977, em que o empreendedorismo representa evidente importância. (IBGE, 20I8). Estando o estado dividido em nove macrorregiões de desenvolvimento econômico, optou-se, por três municípios, considerados com ambiência propicia para o empreendedorismo: Campo Grande (capital do estado), Três Lagoas e Dourados, cidades polos de desenvolvimento econômico e social e onde se encontram os cursos e as universidades selecionadas. As universidades incluem o ensino de empreendedorismo em seus currículos, tanto nos cursos de Administração como de Engenharia de Produção.

Desde o início convém lembrar que este estudo se compõe de duas partes 
que se complementam. Primeiramente, foi mensurada a intenção empreendedora destes alunos, por meio do EIQ, proposto por Liñán, Urbano e Guerrero (2011), com base no que sugere Ajzen (199I). Em seguida, na segunda parte, foi testado o papel mediador da atitude pessoal dos alunos em relação à Intenção Empreendedora, no que se refere às relações com as Normas Subjetivas e com o Controle do Comportamento Percebido, sendo proposto um modelo que amplia o entendimento sobre intenção empreendedora.

$O$ artigo foi estruturado em cinco partes, sendo esta a introdução inicial. A revisão de literatura se volta para a intenção empreendedora, a Teoria do Comportamento Planejado (TCP), e o instrumento EIQ. O método de pesquisa, as técnicas de investigação adotadas e os procedimentos estatísticos para análise dos dados constam a seguir. Os resultados quanto aos objetivos específicos são analisados na quarta parte do estudo. A conclusão retoma os itens considerados importantes na pesquisa, apresentando as limitações do estudo e as sugestões de novas abordagens para a continuidade dos estudos em intenção empreendedora. Complementando se encontram as referências do material citado.

\section{REVISÃO DE LITERATURA}

Existem referências que embasam a intenção empreendedora como obras significativas para o estudo do tema. Estas apresentam os fundamentos da intenção empreendedora e dos modelos de mensuração, alicerçando o assunto em suas significâncias. Se constituem em arcabouços para o conhecimento do tema.

Nesta trajetória, a TCP de Ajzen (1991) se destaca, e deriva da teoria anterior de- nominada Teoria da Ação Fundamentada (TAF), amparada em Fishbein e Ajzen (1975) e Ajzen e Fishbein (1980). Continuando, Ajzen (1985, 1991) buscou explicar o comportamento humano diante de uma situação particular de intencionalidade. 0 modelo de Ajzen (1991) explicou as três variáveis independentes que precedem a formação da intenção e que, por sua vez, preveem o comportamento. Ajzen (1991) argumentou que as atitudes pessoais se referem à atitude ou crença perante um comportamento e correspondem à avaliação favorável ou desfavorável que o indivíduo faz desse comportamento. As crenças normativas influenciam as normas subjetivas, que também refletem na intenção e no comportamento. As crenças de controle influenciam o controle comportamental percebido, que também reflete na intenção e comportamento. Porém entre intenção e comportamento há um controle de comportamento real. De acordo com essa teoria, analisando a intenção empreendedora de um indivíduo, é possível prever se este criará um negócio no futuro.

As obras Ajzen (2002a, 2002b, 20I 2) dedicadas ao mesmo assunto complementam - tema e merecem destaque. A obra de 2002 (Ajzen, 2002a) foi revisada em 2006, e apresenta a construção de um questionário para mensurar a intenção, podendo ser adaptado à necessidade do objeto de estudo. A outra obra de 2002 (Ajzen, 2002b), que apresenta conceitos básicos e a teoria da TCP, foi disponibilizada em linha, em juIho de 2006. Continuando, em 2012, o assunto foi retomado em forma de capítulo de livro (Ajzen, 20I2), revelando a importante contribuição para a TPB, como um modelo de ação racional. Esse estudo discutiu a base conceitual da TCP, sua história 
intelectual e a pesquisa que a gerou. Essas leituras se revestem de importância para o entendimento da teoria postulada por $\mathrm{Aj}$ zen (1985,1991, 2002, 2012), ao longo do tempo. Novamente aqui se realça que estes trabalhos são clássicos para o entendimento do estudo da TCP, sendo que continuam sendo amplamente citados, e se constituindo em base para estudos atuais no que diz respeito à intenção de empreender.

De forma paralela e complementar cabe dizer que o Modelo do Evento Empresarial (EEM) de Shapero e Sokol (1982) e de Shapero (1984) deve, outrossim, ser mencionado. Como a TCP (AJZEN, I99I), esta teoria também é citada com frequência na literatura de intenção empreendedora. Os autores Schlaegel e Koenig (2014) comparam esses dois modelos, aprofundando o assunto e propondo um modelo integrado para a pesquisa em intenção empreendedora, considerando as semelhanças entre eles e servindo de referência para o assunto. Dentre outros pode-se afirmar que Grégoire, Cornelissen, Dimov et al. (2015), Kautonen, Gelderen e Fink (2015), Lortie e Castogiovanni (20/5), e Liñán e Fayolle (20I5) devem ser citados como referência para amparar entendimento sobre as duas teorias: TCP e EEM. Os estudos de Black (20I2), Valliere (20I5), e Souza (20I5), entre outros, apresentam cronologia de autores e de suas obras, que colaboram para evidenciar as contribuições individuais e significativas para a evolução teórica da IE.

Quanto aos modelos apresentados para mensurar a intenção empreendedora, percebe-se que há ênfase na pesquisa de Liñán e Chen (2009). Essa objetivou descrever propriedades psicométricas da construção de um questionário para mensurar a intenção empreendedora, apresentar as análises de confiabilidade e validade realizadas para avaliar a adequação desse instrumento, e testar a aplicabilidade do modelo de intenção empresarial em diferentes contextos culturais: Espanha e Taiwan. Os respondentes foram estudantes universitários. $O$ estudo de tais autores, entretanto, apresentou alguns possíveis problemas em relação à construção do instrumento de coleta de dados, o EIQ. O problema apontado foi o viés de aquiescência, entendido como tendência dos indivíduos a assinalarem Concorda como declarações em uma escala ou instrumento. Com a continuidade das pesquisas sobre Intenção Empreendedora, tema desenvolvido por Liñán, Urbano e Guerrero (20II), esse problema foi resolvido. Houve proposição de 20 questões para mensurar a IE, conservando as construções centrais da TCP de Ajzen (I99I), ou seja, foi estruturado com escala equilibrada, com questões revertidas e conservando a seguinte estrutura: Intenção Empreendedora, Atitude Pessoal, Percepção do Controle do Comportamento e Normas Subjetivas. Liñán, Urbano e Guerrero (20II) desenvolveram uma pesquisa com esse novo instrumento de medida com o objetivo de mensurar a intenção empreendedora. E identificar alguns dos elementos cognitivos ambientais que pudessem explicar diferenças nas intenções empreendedoras em duas regiões espanholas: Catalunha e Andaluzia. A amostra considerou estudantes universitários. Os resultados confirmam a validação do EIQ revisado e atualizado, em cada região pesquisada. $\bigcirc$ que se observa nesses estudos é que as amostras se concentram nos estudantes do ensino superior, revelando que as universidades, desde logo, assumiram um papel importante nesse contexto de inten- 
ções de empreender. As universidades se tornaram campo de investigação sobre o tema em função do relevante e reconhecido papel que desempenham na sociedade e no desenvolvimento regional.

Com este delineamento foram definidas, inicialmente, cinco hipóteses, tomando como base os construtos constantes na TCP de Ajzen (I99I) e amparados nos estudos de Liñán, Urbano e Guerrero (20I I). Da mesma forma nos estudos de Naia (20I3), Liñán, Nabi e Krueger (20I3), Souza (20I5), Souza e Silveira (20I5); Pfeifer, Šarlija e Zekić Sušac (20I6).

\section{HIPÓTESES DA PESQUISA}

HI:A Atitude Pessoal terá relação positiva e significante com a Intenção Empreendedora.

H2: O Controle do Comportamento Percebido terá relação positiva e significante com a Intenção Empreendedora.

H3:As Normas Subjetivas terão relação positiva e significante com a Intenção Empreendedora.

H4: As Normas Subjetivas terão relação positiva e significante com a Atitude Pessoal.

H5:As Normas Subjetivas terão relação positiva e significante com o Controle do Comportamento Percebido.

Com o intuito de ampliar o entendimento do tema, nessa mesma perspectiva, foi realizada a testagem do papel mediador da atitude pessoal dos discentes pesquisados em relação à Intenção Empreendedora no que se refere às relações com as Normas Subjetivas e com o Controle do Comportamento Percebido. Para tanto, foram construídas mais três hipóteses: $\mathrm{H} 6, \mathrm{H} 7$ e $\mathrm{H} 8$. Essa parte do estudo teve ainda amparo em Liñán, Urbano e Guerrero (20II),
Liñán, Nabi e Krueger (20I3), Naia (20I3), Souza (2015) e Souza e Silveira (2015), Pfeifer, Šarlija e Zekić Sušac (2016).

H6: O Controle do Comportamento Percebido terá uma relação positiva e significante com a Atitude Pessoal.

H7:A Atitude Pessoal terá papel mediador na relação entre o Controle do Comportamento Percebido e a Intenção Empreendedora.

H8: A Atitude Pessoal terá papel mediador na relação entre as Normas Subjetivas e a Intenção Empreendedora.

\section{MÉTODO DE PESQUISA}

A pesquisa, de cunho positivista, e segue - ditame de que uma teoria é correta se for passível de comprovação, com métodos científicos validados. Os procedimentos metodológicos da pesquisa foram descritivos e quantitativos, na medida em que essa visa observar as relações entre os fenômenos, adotando recursos e técnicas estatísticas que visem quantificar os dados coletados. Se caracteriza como estudo transversal em que se busca observar sua formação em um momento específico (SELLTIZ;WRIGHTSMAN; COOK, 1987; COOPER;SCHINDLER, 2003; SILVA; LOPES; BRAGA JÚNIOR, 20।4). Para tanto, o método possui caráter inferencial. A amostra foi composta por estudantes universitários dos cursos de Administração e Engenharia de Produção de duas instituições de ensino superior: a Universidade Federal de Mato Grosso do Sul (UFMS) e a Universidade Federal da Grande Dourados (UFGD), as quais desenvolvem conteúdo programático sobre empreendedorismo em disciplinas nos cursos em questão. Esta amostra da pesquisa foi definida como não probabilística, buscando oportunizar que todos os elementos da amostra pudessem fazer parte da pesquisa, 
de forma voluntária. A adoção dessa modalidade de amostra possibilitou, dessa forma, uma participação espontânea dos alunos. Obteve-se o total de 505 respondentes que aderiram responder a pesquisa, no momento da coleta de dados, representando $48,71 \%$ do total da amostra, nas duas universidades, $e$ nos dois cursos.

O instrumento de coleta de dados tomou como base o exposto nos estudos de Liñán, Urbano e Guerrero (20II), Liñán, Nabi e Krueger (20I3), Souza (20I5) e Souza e Silveira (20I5).Apresenta 20 questões, estruturadas em quatro blocos, sendo que as questões apresentam certo grau de liberdade para as respostas, variando de I (discordo plenamente) a 7 (concordo plenamente), em uma escala do tipo Likert.

Para a análise dos dados coletados, os procedimentos de análises foram definidos considerando o uso do software Microsoft Excel 2007, eliminando-se duplicidades de respostas em todos os itens. Para a análise estatística foi utilizado o software SPSS (Statistical Package for Social Sciences) versão I5.0, referente às análises de estatística descritiva e de testes multivariados. Para a análise fatorial confirmatória (AFC) utilizou-se o software Smart PLS 2.0 M3 (RINGLE;WENDE; WILL, 2005), primeiramente, e a Structural Equation Modeling (SEM), conforme Ringle, Silva e Bido (20l4), em seguida. Esses procedimentos são adotados em situações em que o pesquisador das ciências sociais aplicadas se depara com questões de pesquisa que representam fenômenos em modelos "[...] com suporte teórico pouco consagrado ou ainda pouco explorado [...]" (RINGLE, SILVA, BIDO, 20I4, p.55). E quando é recomendado o uso de SEM baseado em variância ou mínimos quadrados parciais (PLS), conforme Hair Junior et al. (20l4). Também se entende, segundo Hair Júnior et al. (2005), que o uso desta abordagem é indicado quando se observa uma distribuição de dados não aderente à normalidade, e ainda quando o objetivo do estudo for buscar uma maior predição das variáveis dependentes.

De maneira complementar, outros testes estatísticos foram utilizados, cabendo destaque para a análise de mediação.A mediação representa um relacionamento indireto e significante entre duas variáveis e que pode ser observado por meio de uma terceira variável. Ou seja, o relacionamento entre as variáveis $\mathrm{X}$ e $\mathrm{Z}$ ocorre por meio do relacionamento de ambas com a variável Y (PREACHER; HAYES, 2004). Para observação da mediação usa-se o teste de Sobel, observando-se os coeficientes de caminho e seus respectivos erros-padrão. Da mesma forma que na moderação, a mediação será significante se $o$ teste $t$ de student retornar um valor significante (SOBEL, 1982).

\section{RESULTADOS DA PESQUISA}

Os resultados quanto às primeiras cinco hipóteses ( $\mathrm{HI}, \mathrm{H} 2, \mathrm{H} 3, \mathrm{H} 4$ e H5) foram confirmados nesta primeira etapa da pesquisa; eles validaram o modelo de escala

QUADRO 1 - Delineamento do instrumento de coleta de dados

\begin{tabular}{|l|l|}
\hline Construto & Escala psicométrica \\
\hline Intenção Emprendedora & A4, A6, A9 - revertida, A13, A17 e A19 - revertida \\
\hline Atitude Pessoal & A2 - revertida, A10, A12 - revertida, A15 e A18 \\
\hline Controle do Comportamento Percebido & A1, A5 - revertida, A7, A14, A16 - revertida, A20 \\
\hline Normas Subjetivsa & A3, A8, A11 \\
\hline
\end{tabular}

Fonte: Dados de pesquisa, 2017. 
psicométrica revista e ajustada por Liñán, Urbano e Guerrero (20II), sendo semeIhante ao encontrado nos estudos de Naia (20 I 3), Liñán, Nabi e Krueger (20 I3), Souza (20I5), Souza e Silveira (20I5), Souza, Silveira e Nascimento (2017).

O modelo apresentou relações positivas e significantes $(p<0,10$ e $t>1,67)$ entre todos os construtos identificados pelo método de reamostragem bootstrap, explicando I3\% da variância da Atitude Pessoal, 21,2\% das Normas Subjetivas e 57,3\% da Intenção Empreendedora. Observou-se que o construto Atitude Pessoal é forte e tem papel significante no modelo e, ainda, que todas as questões foram validadas, indicando que existe intenção dos estudantes em empreender. $O$ modelo final apresentou uma boa qualidade de ajuste do modelo no contexto das instituições de ensino pesquisadas. $O$ cálculo do GoF, proposto por Tenenhaus, Vinzi, Chatelin et al. (2005), por meio da média geométrica entre os valores médios do $R^{2}$ e da AVE média, ficou em 0,440 , ou seja, está de acordo com a recomendação de 0,36 como valor mínimo para as ciências sociais (WETZELS; ODEKERKEN-SCHRÖDER; OPPEN, 2009). Assim, todas as hipóteses foram confirmadas, e a relação entre a Atitude Pessoal e a Intenção Empreendedora $\quad\left(\Gamma=0,612, \mathrm{t}_{(999)}=16,452\right.$, $\mathrm{p}<0,00 \mathrm{I}$ ) demonstrou a maior força entre as relações observadas, denotando o papel consolidado da relação teórica observada entre as atitudes e intenções.

Os indicadores de acurácia do modelo ajustado ou validade preditiva $\left(\mathrm{Q}^{2}\right)$ e tamanho do efeito ou utilidade para a construção do modelo $\left(\mathrm{f}^{2}\right)$ se mostraram satisfatórios. A variável com maior poder de explicação no modelo foi a Atitude Pessoal, com $\mathrm{f}^{2}=0,408$, seguida pelas Normas Subjetivas, com $\mathrm{f}^{2}=0,220$, muito similar ao Controle do Comportamento Percebido, que apresentou $f^{2}=0,207$. A Atitude Pessoal explica a Intenção Empreendedora, como esperado. A variável mais explicada no modelo foi a Intenção Empreendedora $\left(Q^{2}=0,309, R^{2}=0,573\right)$. A relevância preditiva do modelo observada por meio do $\mathrm{Q}^{2}$ evidencia como os coeficientes de caminho mensuram determinada variável. Um maior poder de explicação dessa variável em relação às demais se deve ao fato de que a Intenção Empreendedora antecede o comportamento e reflete que os estudantes universitários integrantes da amostra demonstram predisposição elevada para empreender. Nas características desses estudantes universitários se evidenciam os antecedentes das Normas Subjetivas, que dizem respeito ao reconhecimento de empreendedores na família (pai/mãe/irmãos/ tios/avós/primos) e ao interesse pela participação em algum tipo de capacitação em empreendedorismo.

Esses resultados complementam o que tem sido realizado, geralmente, nos estudos que mensuram a IE, a exemplo de Naia (20I3), Kautonen, Gelderen e Fink (20I5), Souza (20I5), Souza e Silveira (20I6), Pfeifer, Šarlija e Zekić Sušac (20।6), Nabi et al. (2016), Al-Jubari, Hassan e Hashim (2017), Utami (2017), Mwiya (2017), Souza, Silveira e Nascimento (2017), e Zampetakis et al., (2018), dentre outros.

$\mathrm{Na}$ segunda parte da pesquisa, os resultados em relação às hipóteses $\mathrm{H} 6, \mathrm{H} 7$ e $\mathrm{H} 8$ foram testados para 0 modelo estrutural proposto, e verificou-se que o modelo proposto apresentou AVE superior ao mínimo exigido, indicadores de confiabilidade composta e comunalidade adequados, além de GoF satisfatório. Esse resultado pode ser visualizado na Tabela I. 
A Tabela 2 apresenta os indicadores de validade discriminante do modelo estrutural, com a comparação da raiz quadrada das AVEs de cada construto versus a correlação com os demais construtos do modelo. A análise discriminante permite verificar que as variáveis do modelo mensuram seus respectivos construtos.

Os indicadores de acurácia do modelo ajustado ou validade preditiva $\left(\mathrm{Q}^{2}\right)$ e tamanho do efeito ou utilidade para a construção do modelo $\left(f^{2}\right)$ se mostraram satisfatórios. A variável com maior poder de explicação no modelo foi a Atitude Pessoal (AP) $\left(f^{2}=0,408\right)$; Controle do Comportamento percebido (CCP) $\left(f^{2}=0,207\right)$ e Normas Subjetivas (NS) $\left(f^{2}=0,220\right)$ aparecem na sequência. A variável mais explicada foi a Intenção Empreendedora (IE) $\left(\mathrm{Q}^{2}=0,309\right)$, segui- da da Atitude Pessoal (AP) $\left(Q^{2}=0,174\right)$ e de Normas Subjetivas (NS) $\left(\mathrm{Q}^{2}=0,107\right)$.

As relações positivas e significantes $(p<0,10$ e $t>1,67)$ estiveram presentes entre todos os construtos, identificados pelo método de reamostragem bootstrap, explicando $30,5 \%$ da variância da Atitude Pessoal (AP), 2I,2\% das Normas Subjetivas (NS) e 57,3\% da Intenção Empreendedora (IE).

Para o modelo proposto, em seu entendimento integrado, observou-se relação significante entre: Atitude pessoal e Intenção empreendedora $(\mathbf{H I}, \Gamma=0,6 \mid 4$, $\left.\mathrm{t}_{(999)}=15,777, \mathrm{p}<0,00 \mathrm{I}\right)$; Controle do comportamento percebido e Intenção empreendedora $\left(\mathbf{H 2}, \Gamma=0,173, \mathrm{t}_{(999)}=4,399\right.$, $\mathrm{P}$-value $<0,00 \mathrm{I})$; Normas subjetivas e Intenção empreendedora $(\mathbf{H} 3, \Gamma=0,077$, $\mathrm{t}_{(999)}=2,157, \mathrm{p}$-value=0,032), Normas sub-

TABELA 1 - Indicadores de ajuste, validade convergente e confiabilidade

\begin{tabular}{l|c|c|c|c|c|c}
\hline Construto & AVE & $\begin{array}{c}\text { Confiabilidade } \\
\text { composta }\end{array}$ & $\mathbf{R}^{\mathbf{2}}$ & $\begin{array}{c}\text { Alpha de } \\
\text { Cronbach }\end{array}$ & Comunalidade & Redundância \\
\hline Atitude pessoal & 0,606 & 0,884 & 0,307 & 0,836 & 0,606 & 0,171 \\
\hline Controle do comportamento percebido & 0,515 & 0,808 & - & 0,685 & 0,515 & - \\
\hline Intenção empreendedora & 0,577 & 0,845 & 0,574 & 0,755 & 0,577 & 0,302 \\
\hline Normas subjetivas & 0,603 & 0,820 & 0,212 & 0,671 & 0,603 & 0,128 \\
\hline
\end{tabular}

Fonte: Dados de pesquisa, 2017.

Nota: $\mathrm{GoF}=0,469$, Média das AVEs=0,575, Média R2=0,364

TABELA 2 - Indicadores de validade discriminante

\begin{tabular}{l|c|c|c|c}
\hline Construto & $\begin{array}{c}\text { Atitude } \\
\text { pessoal }\end{array}$ & $\begin{array}{c}\text { Controle do comportamento } \\
\text { percebido }\end{array}$ & $\begin{array}{c}\text { Intenção } \\
\text { empreendedora }\end{array}$ & $\begin{array}{c}\text { Normas } \\
\text { subjetivas }\end{array}$ \\
\hline Atitude pessoal & $\mathbf{0 , 7 7 8}$ & - & - & - \\
\hline $\begin{array}{l}\text { Controle do comportamento } \\
\text { percebido }\end{array}$ & 0,539 & $\mathbf{0 , 7 1 8}$ & - & - \\
\hline Intenção empreendedora & 0,735 & 0,540 & $\mathbf{0 , 7 5 9}$ & - \\
\hline Normas subjetivas & 0,360 & 0,461 & 0,378 & $\mathbf{0 , 7 7 6}$ \\
\hline
\end{tabular}

Fonte: Dados de pesquisa, 2017.

Nota: Valores em negrito representam a raiz quadrada das AVEs. 
jetivas e Atitude pessoal $(\mathbf{H 4}, \Gamma=0,142$, $\mathrm{t}_{(999)}=2,976, \mathrm{p}$-value=0,003); e Normas subjetivas e Controle do comportamento percebido $\left(\mathbf{H} 5, \Gamma=0,46 \mid, t_{(999)}=12,288\right.$, $p$ value $<0,00 \mathrm{I}$ ). $\mathrm{E}$, ainda, há a integração de mais três hipóteses:

I) a relação entre Controle do comportamento percebido e Atitude pessoal $(\mathbf{H 6}$, $\Gamma=0,474, t_{(999)}=1 \mathrm{I}, 580$, p-value $\left.<0,00 \mathrm{I}\right)$;

2) a mediação $(\mathbf{H 7})$ da Atitude pessoal para as relações entre Controle do comportamento percebido e Intenção empreendedora (Teste de Sobel=9,43I , $\mathrm{p}<0,00 \mathrm{I}$ );

3) a mediação (H8) da Atitude pessoal para a relação entre Normas subjetivas e Intenção empreendedora (Teste de Sobel=2,92I, $p=0,003$ ).
A análise estrutural indicou caminhos significativos entre os construtos finais do modelo integrado, conforme Tabela 3.

A análise estrutural indicou caminhos significativos também entre os construtos do modelo, e por meio dela se verifica a confirmação de todas as hipóteses.

O modelo proposto para a mensuração da intenção empreendedora, com comprovação integrada de oito hipóteses, nas quais se inclui a mediação da Atitude Pessoal em relação à Intenção Empreendedora no que tange às relações com as Normas Subjetivas e com o Controle do Comportamento Percebido, pode ser visualizado na Figura I, com a mediação representada pelas setas pontilhadas.

TABELA 3 - Indicadores estruturais finais

\begin{tabular}{|c|c|c|c|c|c|c|c|}
\hline Hipótese & Relação & 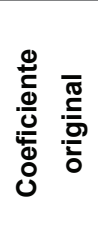 & 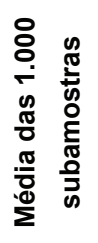 & $\begin{array}{l}\text { Desvio } \\
\text { padrão }\end{array}$ & Teste $\mathbf{t}$ & $\mathrm{p}$-valor & Resultado \\
\hline $\mathrm{H} 1$ & Atitude pessoal $\downarrow$ Intenção empreendedora & 0,614 & 0,616 & 0,039 & 15,777 & 0,001 & Confirmada \\
\hline $\mathrm{H} 2$ & $\begin{array}{l}\text { Controle do comportamento percebido } \downarrow \text { Inten- } \\
\text { ção empreendedora }\end{array}$ & 0,173 & 0,171 & 0,039 & 4,399 & 0,001 & Confirmada \\
\hline $\mathrm{H} 3$ & Normas subjetivas $\downarrow$ Intenção empreendedora & 0,077 & 0,078 & 0,036 & 2,157 & 0,032 & Confirmada \\
\hline $\mathrm{H} 4$ & Normas subjetivas $\downarrow$ Atitude pessoal & 0,142 & 0,142 & 0,048 & 2,976 & 0,003 & Confirmada \\
\hline H5 & $\begin{array}{l}\text { Normas subjetivas } \downarrow \text { Controle do comportamen- } \\
\text { to percebido }\end{array}$ & 0,461 & 0,463 & 0,038 & 12,288 & 0,001 & Confirmada \\
\hline $\mathrm{H} 6$ & $\begin{array}{l}\text { Controle do comportamento percebido } \downarrow \text { Ati- } \\
\text { tude pessoal }\end{array}$ & 0,474 & 0,478 & 0,041 & 11,580 & 0,001 & Confirmada \\
\hline $\mathrm{H} 7$ & $\begin{array}{l}\text { Controle do comportamento percebido*Ati- } \\
\text { tude*Intenção empreendedora }\end{array}$ & & - & - & 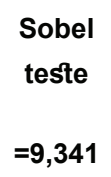 & 0,001 & Confirmada \\
\hline H8 & $\begin{array}{l}\text { Normas subjetivas }{ }^{*} \text { Atitude*Intenção em- } \\
\text { preendedora }\end{array}$ & & - & - & 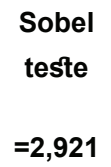 & 0,003 & Confirmada \\
\hline
\end{tabular}

Fonte: Dados de pesquisa, 2017. 


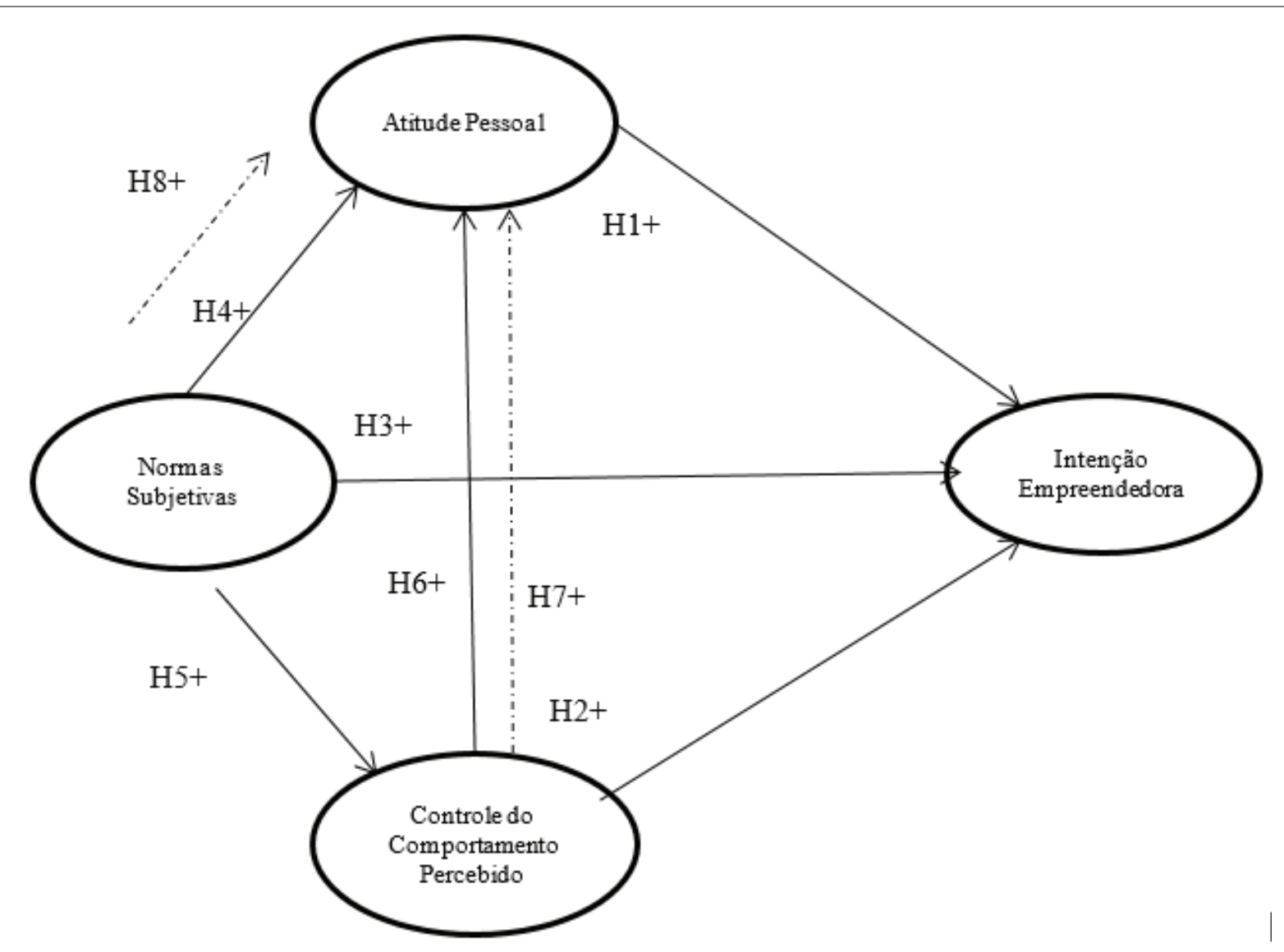

FIGURA 1 - Modelo estrutural proposto

Fonte: Dados de pesquisa, 2017.

Nota: H7 e H8: efeitos mediadores da atitude pessoal (linha pontilhada).

\section{ANÁLISE E DISCUSSÃO DOS RESULTADOS}

A mediação esperada da Atitude Pessoal foi comprovada como antecedente do comportamento humano. Esta indicação demonstra que a alteração proposta no modelo validado por Liñán, Urbano e Guerrero (20ll), adicionando mais três hipóteses que aqui foram testadas e comprovadas, mostrou-se adequada. Assim, em relação às três hipóteses que foram acrescentadas e testadas no modelo estrutural proposto, nesse estudo, as análises estruturais se revelaram significativas para os construtos, conforme se apresenta:

H6: Controle do Comportamento Per- cebido exerce influência nas Atitudes Pessoais; a análise estrutural indicou caminhos significativos entre esses dois construtos, em que se têm os seguintes coeficientes: $\Gamma=0,474$ - indica uma relação muito forte entre os dois construtos; $\mathrm{t}=\mid \mathrm{I}, 580 \mathrm{e}$ $\mathrm{P}<0,00 \mathrm{I}$ - significa que os caminhos entre os construtos são significantes; CCP exerce influência significativa em AP.

H7: a Atitude Pessoal tem um papel mediador para a relação entre o Controle do Comportamento Percebido e a Intenção Empreendedora. A relação entre uma variável CCP e uma variável IE sofre influência de uma variável AP, o que indicou caminhos significativos entre esses dois constru- 
tos; foi realizado o Teste de Sobel=9,43I, $\mathrm{p}<0,00$ I.

H8: A Atitude Pessoal tem um papel mediador entre as Normas Subjetivas e a Intenção Empreendedora. A relação entre uma variável NS e uma variável IE sofre influência de uma variável $A P$, o que indicou caminhos significativos entre esses dois construtos. Foi realizado o Teste de Sobel $=2,921, p=0,003$.

A atitude pessoal se fortalece em 17,5\% a mais no modelo proposto se comparado ao modelo inicial. Cabe destacar que a Atitude Pessoal é a variável proposta por Ajzen (I99I) com maior influência no modelo proposto. O Controle do Comportamento Percebido e a Intenção Empreendedora apresentam percentuais idênticos no modelo inicial de Liñán, Urbano e Guerrero (20l I), bem como no modelo proposto neste estudo. Este resultado, que comprova a Atitude Pessoal como variável de maior influência, amplia e reforça o que vem sendo evidenciado em estudos nos quais a TCP (Ajzen, 199l) deu suporte para as escalas psicométricas utilizadas, explicando as relações entre os construtos, e representou um passo importante na análise de variáveis e na validação na escala psicométrica EIQ (Liñán, Urbano e Guerrero, 20II) de previsão das intenções empreendedoras.

A reflexão que se faz sobre esse resultado é que ser empreendedor reside muito mais na atitude pessoal de responsabilidade do indivíduo. $E$ esta responsabilidade parece estar presente nos alunos dos cursos de graduação pesquisados, visto que vem sendo despertada e construída com base em conteúdos ministrados nos cursos de graduação em Administração e Engenharia de Produção nas duas universidades federais do Estado de Mato Grosso do Sul.
Estas apresentaram papel relevante perante a sociedade sul-mato-grossense, tendo ênfase e enfoque no desenvolvimento das competências empreendedoras dos seus acadêmicos. Verificou-se que a UFMS teve todas as hipóteses e relações confirmadas para os dois cursos nas duas unidades objeto da pesquisa. Tanto no Curso de Administração quanto no de Engenharia de Produção da UFGD, não foi confirmada apenas a relação entre os construtos Normas Subjetivas e Intenção Empreendedora. No modelo estrutural proposto, as relações entre tais construtos são as que menos se confirmam.

Segundo Liñán e Chen (2009), Normas Subjetivas é o construto que mede a pressão social. As crenças normativas influenciam as normas subjetivas, que também refletem na intenção e comportamento. Liñán, Urbano e Guerrero (20II) sinalizaram que em outros estudos não se encontrou nenhuma relação direta e significativa entre normas subjetivas e intenção empreendedora; os estudos de Naia (2013) seguiram o mesmo resultado. Como as normas subjetivas dizem respeito às influências do contexto, a sua baixa significância pode ser entendida como influência de um contexto de recessão desfavorável. Podese vislumbrar ainda considerações sobre - ambiente político, econômico e cultural para justificar a fragilidade do construto no modelo. A baixa influência das normas subjetivas pode representar, da mesma forma, uma sociedade no início do desenvolvimento de uma cultura empreendedora. Jaén e Liñán (20/3) indicaram a conveniência de incluir no modelo e na escala psicométrica medidas para verificar o nível da cultura empreendedora existente na região em consonância com as Normas Subjetivas. 
As hipóteses para a ampliação do modelo apresentado por Liñán, Urbano e Guerrero (20I I) foram construídas considerando: H6: o Controle do comportamento percebido teria relação positiva e significante com a Atitude pessoal; H7: a Atitude pessoal teria um papel mediador para a relação entre o Controle do comportamento planejado e a Intenção empreendedora; H8: a Atitude pessoal teria um papel mediador para a relação entre as Normas subjetivas e a Intenção empreendedora.

Neste estudo as três hipóteses foram confirmadas. $O$ construto Atitude Pessoal, tanto no presente estudo quanto no de Liñán, Urbano e Guerrero (20l I) teve o maior poder de explicação. No presente estudo, no modelo estrutural proposto, fortaleceu-se em 17,5\% a mais, comprovando que a Atitude Pessoal é o construto de maior influência na Intenção empreendedora. Deixa entrever que a intenção empreendedora como antecedente imediato do comportamento. Os determinantes da atitude pessoal, norma subjetiva e controle comportamental percebido seguem as crenças sobre as prováveis consequências do comportamento, sobre as expectativas normativas e sobre a presença de fatores que controlam o desempenho c o mportamental. Esse resultado ass emelha-se ao encontrado no estudo de Souza, Silveira e Nascimento (2016).

\section{CONCLUSÃO}

Se pode concluir que o tema Intenção Empreendedora consiste em estudar o comportamento humano, fundamentado aqui na Teoria do Comportamento Planejado (TCP) de Ajzen (I99I), a qual é reconhecida e considerada a teoria dominante, principalmente analisando-se os estudos desenvolvidos por Schlaegel e Koenig (20I4), Lortie e Castogiovanni (20I5) e Liñán e Fayolle (20I5). Nessa perspectiva, e com base na TPB (Ajzen, 1991), os pesquisadores Liñán e Chen (2009) aplicaram uma escala psicométrica denominada EIQ para verificar o grau de intenção de empreender. Liñán, Urbano e Guerrero (20II) revisaram e atualizaram este EIQ, aprimorando a escala. Seguindo essa linha, o tema mostrou-se relevante para ser desenvolvido em um contexto regional, com o intuito de contribuir para o aperfeiçoamento do modelo e validando esta escala psicométrica em contextos ainda inexplorados.

A presente pesquisa, em sua primeira parte, validou a escala psicométrica revista e ajustada por Liñán, Urbano e Guerrero (20II) na UFMS e na UFGD, onde as primeiras cinco hipóteses $(\mathrm{HI}, \mathrm{H} 2, \mathrm{H} 3, \mathrm{H} 4, \mathrm{H} 5)$ foram aceitas. $\mathrm{Na}$ segunda parte da pesquisa, foram testadas mais três hipóteses $(H 6, H 7, H 8)$, evidenciando que a mediação esperada para a Atitude Pessoal foi comprovada. Esta variável se revelou forte e superior quanto às demais: Normas Subjetivas e Controle do Comportamento Percebido, e se apresentou como antecedente do comportamento humano. Aqui, nesta testagem, a atitude pessoal se fortaleceu em $17,5 \%$ a mais do que no modelo validado por Liñán, Urbano e Guerrero (20II) na primeira fase desta pesquisa, o que indica que a ampliação de tal modelo mostrouse adequada. Esta conclusão permitiu propor a ampliação do modelo de Liñán, Urbano e Guerrero (20II) quanto à mensuração da Intenção Empreendedora. 
Por sua vez, com a aceitação e comprovação das três hipóteses testadas na segunda parte do estudo, pode-se afirmar que elas reforçaram e ampliaram o entendimento de que as crenças comportamentais induzem a uma atitude pessoal, favorável ou desfavorável, quanto à ação de empreender. Assim, este estudo permitiu aprofundar a análise empírica de variáveis consideradas antecedentes do comportamento empreendedor, notadamente a Atitude Pessoal. Esta conclusão vem acrescentar conhecimentos na literatura do tema Intenção Empreendedora, permitindo ir além do que foi apresentado nos estudos que embasaram o aqui desenvolvido, sendo o de Liñán e Chen (2006), Liñán, Urbano e Guerrero (20II), Liñán, Nabi e Krueger (20I3) e Souza e Silveira (20I6).

A metodologia adotada para os procedimentos de coleta e de análise dos dados mostrou-se adequada para a realização da pesquisa, espelhando-se no que vem sendo adotado e preconizado em estudos semeIhantes e que embasaram o assunto, revisados e citados ao longo desse estudo.

Avaliando os resultados obtidos com este estudo, é possível afirmar que a questão de pesquisa foi respondida afirmativamente e que os objetivos foram atingidos. $O$ modelo estrutural proposto foi delineado, e, dessa forma, pode-se afirmar que esta pesquisa abre possibilidades para novos estudos e reflexões para o fortalecimento da pesquisa científica na área de intenção empreendedora, tendo contribuído para: validar uma escala psicométrica para mensurar a in- tenção empreendedora no contexto de duas universidades brasileiras; comprovar que a escala psicométrica ajustada por Liñán, Urbano e Guerrero $(20 \mathrm{II})$ reafirma a teoria de base de Ajzen (1991) e prediz a propensão de estudantes de ensino superior de universidades federais brasileiras de se tornarem empreendedores; ampliar a mensuração dos construtos propostos por Liñán, Urbano e Guerrero (20II) com mais três hipóteses que reforçam o entendimento sobre a intenção empreendedora; e propor um modelo estrutural que amplia o entendimento da Intenção Empreendedora no que se refere ao papel mediador da atitude pessoal dos estudantes universitários de universidades federais brasileiras, em cursos de graduação, em relação à Intenção Empreendedora, quanto às relações com as Normas Subjetivas e o Controle do Comportamento Percebido.

Cabe apontar algumas limitações da pesquisa, as quais estão relacionadas ao fato de serem consideradas somente duas universidades federais do Mato Grosso do Sul, em detrimento das demais universidades federais, públicas, em nível estadual e particular existentes. Entretanto, este fato se justifica em razão da acessibilidade e concordância por parte dos dirigentes e da disponibilidade dos estudantes universitários em participar da pesquisa. Este fato não invalida o que aqui foi apresentado, uma vez que as duas universidades são tradicionais, consideradas de excelência e relevantes para o Estado de Mato Grosso do Sul, permitindo um estudo regional. E, ainda, é sempre possível ampliar a pesquisa, realizando um novo estudo, em um momento mais favo- 
rável e em outro ambiente, permitindo, inclusive, a comparação ao longo do tempo. Cabe apontar, ainda, como limitação o fato de a amostra não ter sido probabilística, como esperado para estudos quantitativos. Justifica-se o delineamento adotado em razão de que quase $50 \%$ dos alunos de graduação, dos dois cursos, aderiram e responderam, de forma voluntária, a esta pesquisa. Assim, o número de respondentes ampara a escolha da amostra.

Concluindo, pode-se afirmar que esta pesquisa oportunizou ampliar a temá- tica da intenção empreendedora no que diz respeito ao relacionamento de variáveis do constructo apresentado por Liñán, Urbano e Guerrero (20II), amparado no estudo seminal de Ajzen (1991). É possível afirmar, ainda, que com esta ampliação a proposição de modelo integrado complementar abre novas possibilidades para estudos complementares e reflexões para o fortalecimento da pesquisa científica na área de intenção empreendedora. 
AJZEN, I. Constructing a TpB questionnaire: conceptual and methodological considerations. Sept. 2002a (Revised January, 2006). Disponível em: https://pdfs. semanticscholar.org/0574/b20bd58 I 30dd5a96 I f la2db I Ofd I fcbae95d.pdf Acesso em: 25 Apr.20I8. . From intentions to actions: a Theory of Planned Behavior. In: KUHL, J.; BECKMAN, J. (Ed.). Action-control: from cognition to behavior. Heidelberg: Springer. 1985. p. II-39.

. Perceived behavioral control, self-efficacy, locus of control and the Theory of Planned Behavior. Journal of Applied Social Psychology, v.32, i.4, p.665-683, April 2002b. 3I July 2006 issue Online. Disponível em: https:// onlinelibrary.wiley.com/doi/ abs/I0.IIIII/j.I559-I8I6.2002. tb00236.xAcesso em:25 abr.20।8. The Theory of Planned Behavior. Organizational Behavior and Human Decision Processes, v. 50, i. 2, p. 179 211, 1991.

. The Theory of Planned Behavior. In: LANGE, P. A. M.; KRUGLANSKI, A. W.; HIGGINS, E.T. (Ed). Handbook of theories of social psychology. London: Sage, 20I2. v. I, p. 438-459.

.; FISHBEIN, M. Understanding attitudes and predicting social behavior. Englewood Cliffs: Prentice-Hall, 1980.

AL-JUBARI, I.; HASSAM, A., HASHIM, J. The role of autonomy as a predictor of entrepreneurial intention among university students in Yemen. International Journal of Entrepreneurship and Small Business, v.30, i.3, p.325340, 2017.

BLACK, M. M. Exploring the multi- focus influence of identity on students' entrepreneurial intent. 2012. Disponível em: https:// shareok.org/handle/ I I 244/7204 Acesso em: 12 Feb. 2017.

DEBARLIEV, S. et al. Antecedents of entrepreneurial intention: Evidence from Republic of Macedonia. Business and Economic Horizons, v. I I, i.3, p. I43-16I, 2015. FAYOLLE, A.; LIÑÁN, F. A systematic literature review on entrepreneurial intentions: Citation, thematic analyses, and research agenda. International Entrepreneurship and Management Journal, v. II, i. 4, p. 907-933, 2015. .; MORIANO,

J.A. Beyond entrepreneurial intentions: values and motivations in entrepreneurship. International Entrepreneurship and Management Journal, v. I0, i.4, p.679689, Dec. 2014.

FISHBEIN, M.; AJZEN, I. Belief, attitude, intention and behavior: an introduction to Theory and Research. Reading, MA: Addison -Wesley, 1975.

FONTENELE, R. E. S.; BRASIL, M. V. O.; SOUSA, A. M. R. Influência da Intenção empreendedora de discentes em um instituto de ensino superior. Revista de Empreendedorismo e Gestão de Pequenas Empresas, v.4, n.3, p. |47-176, 2015.

GRÉGOIRE, D. A. et al. The mind in the middle: taking stock of affect and cognition research in entrepreneurship. International Journal of Management Review, v. 17, i. 2, p. 125-142, 2015.

HAIR, J. F. et al. A primer on Partial Least Squares Structural Equation Modeling (PLSSEM). Los Angeles: SAGE, 2014. HAIR JÚNIOR, J. F.;ANDERSON, R. E.;
TATHAM, R. L.; BLACK,W. C. Análise multivariada de dados. 5 . ed. Porto Alegre: Bookman, 2005.

INSTITUTO BRASILEIRO DE GEOGRAFIA E ESTATÍSTICA (IBGE). Dados sócio econômicos do estado de Mato Grosso do Sul. Disponível em: https://cidades.ibge.gov.br/brasil/ms/panorama Acesso em: 24 abril 2018.

KAUTONEN, T.; GELDEREN, M.; FINK, M. Robustness of the theory of planned behaviour in predicting entrepreneurial intentions and actions. Entrepreneurship Theory and Practice, v. 39, i. 3, p. 655-674, 2015.

KIM-SOON, N.; AHMAD, A.R.; IBRAHIM, N.N. Theory of Planned Behaviour: undergraduates' entrepreneurial motivation and entrepreneurship career intention at a public university. Journal of Entrepreneurship: Research \& Practice, v. 2016, Article ID 792385, I4p. Disponível em: http://ibimapublishing.com/articles/JERP/20 I 6/792385/792385. pdf Acesso em: 24 abr. 2018.

KRUEGER, N. F; CARSUD, A. Entrepreneurship intentions: applying the Theory of Planned Behavior. Entrepreneurship \& Regional Development, v. 5, p. 316-323, 1993.

LIÑÁN, F. Skill and value perceptions: how do they affect entrepreneurial intentions? International Entrepreneurship and Management Journal, v. 4, i. 3, p. 257272, 2008.

.; CHEN, Y. Development and cross-cultural application of a specific instrument to measure entrepreneurial intentions. Entrepreneurship Theory and Practice, v. 33, i. 3, p. 593-617, May 2009.

; FAYOLLE, A. A systema- 
tic literature review on entrepreneurial intentions: citation, thematic analyses, and research agenda. International Entrepreneurship and Management Journal, v. II, i. 4, p. 907-933, Dec. 2015.

; NABI, G.; KRUEGER, N. British and Spanish entrepreneurial intentions: a comparative study. Revista de Economía Mundial, v. 33, p. 73-103, 2013. .; URBANO, D; GUERRE-

$R O, M$. Regional variations in entrepreneurial cognitions: start-up intentions of university students in Spain. Entrepreneurship \& Regional Development, v. 23, i. 3-4, p. I87-2 I 5, Apr. 20 I I.

LORTIE, J.; CASTOGIOVANNI, G. The theory of planned behavior in entrepreneurship research: what we know and future directions. International Entrepreneurship and Management Journal, Mar. 2015.

LOVISON, M. Capital social e intenção empreendedora dos estudantes de administração de uma instituição de ensino superior da serra gaúcha. 2014. Dissertação (Mestrado em Administração) -Programa de Pós-Graduação em Administração da Universidade de Caxias do Sul, Caxias do Sul, 20l4.

MWIYA, B. et al. Predicting the entrepreneurial Intentions of university students: applying the Theory of Planned Behavior in Zambia, Africa. Open Journal of Business and Management, v.5, p. 592 $610,2017$.

$\mathrm{NABI}, \mathrm{G}$. et al. Does entrepreneurship education in the first year of higher education develop entrepreneurial intentions? The role of learning and inspiration, Studies in Higher Education, v. 43, i.3, p.452-467, 2016.

NAIA, A. M. P. Entrepreneurship education in sport sciences: implications for curriculum development. 2013. Tese (Doutorado em Ciências da Educação) -Universidade de Lisboa, Lisboa, Portugal, 2013.

PFEIFER, S.; ŠARLIJA, N.; ZEKIC SUŠAC, M. Shaping the Entrepreneurial Mindset: Entrepreneurial Intentions of Business Students in Croatia. Journal of Small Business Management, v. 54, i. I, p. I02-I |7, 2016.

PREACHER, K. J.; HAYES, A. F. SPSS and SAS procedures for estimating indirect effects in simple mediation models. Behavior Research Methods, Instruments \& Computers, v. 36 , i. 4, p. 7 I773I, 2004.

QIAN, S. et al.The relationship between entrepreneurship education and entrepreneurial intentions: a meta-analytic review. Entrepreneurship Theory and Practice, v. 38, i. 2, p. 2 I 7-254, Mar. 20 I4. RINGLE, C. M.; SILVA, D. da; BIDO, D.E. Modelagem de equações estruturais com utilização do SmartPLS. Brazilian Journal of Marketing - BJM. Revista Brasileira de Marketing - ReMark. Edição Especial, v. I3, n. 2, Mai. 2014.

RINGLE, C. M.; WENDE, S.; WILL, A. SmartPLS 2.0 (M3) Beta. Hamburg: University of Hamburg, 2005. Disponível em: <www.smartpls.de>. Acesso em: 27 Jan. 2017.

RUEDA, S.; MORIANO, J. A.; LIÑÁN, F. Validating a theory of planned behavior questionnaire to measure entrepreneurial intentions. In: FAYOLLE, A., KYRÖ, P., LIÑÁN, F. (Ed.). Developing, Shaping and Growing Entrepreneurship. Cheltenham: Edward Elgar, 2015. p. 60-78.

SANTOS, E. B. A.; MARTINS, F. S.; SILVEIRA, A. Intenção empreendedora: categorização, classifica- ção de constructos e predição de modelo. In: ENCONTRO DA ASSOCIAÇÃO NACIONAL DE PÓS-GRADUAÇÃO E PESQUISA EM ADMINISTRAÇÃO ENCONTRO DA ANPAD, 40, Costa do Sauipe, 2016. Anais... Costa do Sauipe:ANPAD, 2016.

SCHLAEGEL, C.; KOENIG, M. Determinants of Entrepreneurial Intent: A Meta-Analytic Test and Integration of Competing Models. Entrepreneurship Theory and Practice, v. 38, i. 2, p. 291-332, 2014.

SELLTIZ, C.; WRIGHTSMAN, L. S.; COOK, S.W. Dados de observação e de arquivo. Métodos de Pesquisa nas Relações Sociais, v. 2, p. 95-I20, 1987.

SHAPERO, A. The entrepreneurial event. In: KENT, C. A. (Ed.). The Environment for Entrepreneurship. Lexington: D.C. Heath \& Company, 1984. p. 21-40. .; SOKOL, L. The social dimensions of entrepreneurship. In: KENC, C. A.; SEXTON, D. L.; VESPER, K. H. (Ed.). Encyclopedia of entrepreneurship. Englewood Cliffs: Prentice-Hall, 1982. p. 72-90.

SILVA, D. da; LOPES, E. L.; BRAGA JUNIOR, S. S. Pesquisa quantitativa: elementos, paradigmas e definições. GeSec - Revista de Gestão e Secretariado, v.5, n.I, p.0I-I8, Jan./Apr. 2014.

SILVEIRA,A. et al. Factors and approaches of entrepreneurial intention. REGEPE - Revista de Empreendedorismo e Gestão de Pequenas Empresas, v. 6, i.2, p. 263-290, 2017.

SOBEL, M. E. Asymptotic intervals for indirect effects in structural equations models. In: LEINHART, S. (Ed.). Sociological methodology. San Francisco: Jossey-Bass, 1982. p. 290-312.

SOUZA, R. S. Intenção empreen- 
dedora: validação de modelo em universidades federais de Mato Grosso do Sul, Brasil. 2015. Tese (Doutorado em Administração) -Programa de Pós-Graduação em Administração, Universidade Nove de Julho, São Paulo, 2015. .; SILVEIRA, A. Intenção

empreendedora: validação do Entrepreneurial Intention Questionnaire (EIQ) em contexto brasileiro. In: ENCONTRO DA ASSOCIAÇÃO NACIONAL DE PÓS-GRADUAÇÃO E PESQUISA EM ADMINISTRAÇÃO, 40, Costa do Sauipe, 2016. Anais... Costa do Sauipe:ANPAD, 2016. ; NASCIMEN-

TO, S. do .Ampliando a mensuração de intenção In: ENCONTRO DA ASSOCIAÇÃO NACIONAL DE PÓS-GRADUAÇÃO E PESQUISA EM ADMINISTRAÇÃO, 4I, São Paulo, SP. 2017. Anais... Rio de Janeiro, RJ,Anpad, 20I7.v. I. p. I-I6.
TENENHAUS, M. et al. Pls Path Modeling. Computational Statistics \& Data Analysis, v. 48, p. I 59-205, 2005.

Terjesen, S; Hessels; j.; Li, d. Comparative international entrepreneurship: a review and research agenda. Journal of Management, v. 42, p. 299-344, 2016.

UTAMI, C.W. Attitude, subjective norms, perceived behavior, entrepreneurship education and self-efficacy toward entrepreneurial intention university student in Indonesia. European Research Studies, v. 20, i.2, p. 475, 2017.

VASCONCELOS,V. N. S.A. et al. Intenção empreendedora de estudantes universitários: estudo sob a perspectiva da Teoria Sócio Cognitiva do Desenvolvimento de Carreira. In: ENCONTRO DA ASSOCIAÇÃO NACIONAL DE PÓS-GRADUAÇÃO E PESQUISA EM ADMINISTRAÇÃO, 4I, São Paulo, SP.
2017. Anais... Rio de Janeiro, RJ, Anpad, 2017.v. I. p. I-I6.

WETZELS, M.; ODEKERKEN-SCHRÖDER, G.; OPPEN, C. V. Using PLS path modeling for assessing hierarchical construct models: guidelines and empirical illustration. MIS Quarterly, v. 33, i. I, p. I77-195, 2009.

ZAMPETAKIS L.A. et al. Gender-based differential item functioning in the application of the Theory of Planned Behavior for the study of entrepreneurial intentions. Frontiers in Psychology. v. 8, p.45I, 2018. Doi: 10.3389/ fpsyg.20I7.0045 I

ZHANG, Y.; DUYSTERS, G. M.; CLOODT, M. M.A. H. The role of entrepreneurship education as a predictor of university students' entrepreneurial intention. International Entrepreneurship and Management Journal, v.10, n.3, p. 623-64I, 2014. 